\title{
Review: evidence is lacking to show that adults given fluids $1.5-3$ hours preoperatively have greater risks of aspiration or regurgitation than those given a standard fast
}

Brady M, Kinn S, Stuart P. Preoperative fasting for adults to prevent perioperative complications. Cochrane Database Syst Rev 2003;(4):CD004423 (latest version 27 Aug 2003).

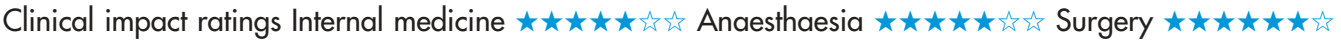

In adults, what are the effects of different preoperative fasting regimens (duration, type, and volume of permitted intake) on perioperative complications and patient wellbeing?

\section{METHODS}

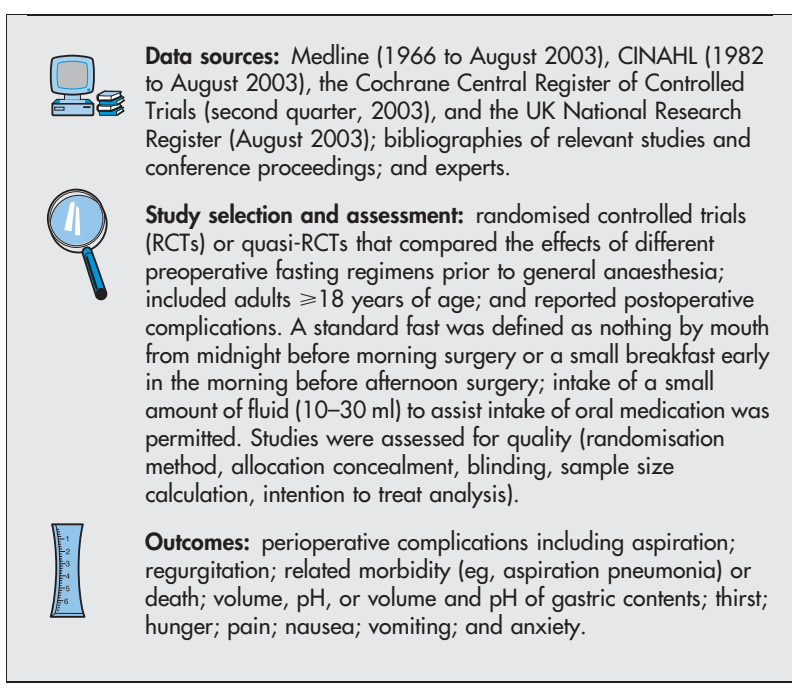

\section{MAIN RESULTS}

22 trials ( 38 RCT comparisons, $\mathrm{n}=2270$ ) met the selection criteria 15 trials reported patients' experience of the fasting process (ie thirst, hunger, pain, nausea, vomiting, and anxiety). Most trials focused on generally healthy patients not thought to be at increased risk of regurgitation or aspiration during anaesthesia, although 3 trials included patients considered to be at a higher risk (obese patients in 1 trial and postpartum women in 2 trials). Trials mainly reported on patients having elective surgery.

In 6 trials that compared a shortened fluid fast (fluids given 1.5-3 $\mathrm{h}$ preoperatively) with a standard fast and recorded aspiration or regurgitation, none reported any aspiration or regurgitation. 24 comparisons examined a shortened fluid fast versus a standard fast and found no differences in volume or $\mathrm{pH}$ of gastric contents. Fluids permitted preoperatively included water, coffee, fruit juice, clear fluids, and other drinks. In 9 comparisons, patients given a drink of For correspondence: Dr M Brady, Nursing Research Initiative for Scotland, Glasgow, UK. m.brady@gcal.ac.uk

Source of funding: Chief Scientist Office, Scottish Executive Health Department, UK. water preoperatively $(n=319)$ had a lower volume of intraoperative gastric contents than those who had a standard fast $(n=292)$ (weighted mean difference $-6.16 \mathrm{ml}, 95 \% \mathrm{CI}-9.41$ to -2.91 ). 24 comparisons examined different volumes of preoperative intake (ie, low, medium, high, or unlimited) versus a standard fast and found no differences in perioperative outcomes.

\section{CONCLUSION}

Evidence is lacking that adults given fluids 1.5-3 hours preoperatively have a greater risk of aspiration or regurgitation than those who have a standard fast.

\section{Commentory}

G astric regurgitation, pulmonary aspiration, and subsequent pneumonitis are feared complications during anaesthesia. The current risk of aspiration is estimated to be 3 per 10000 in patients who are healthy and is 3-4 times higher in patients in obstetrics or emergency surgery. ${ }^{\prime}<20 \%$ of aspirations result in serious morbidity or mortality. ${ }^{\prime}$ The relation between the volume and acidity of the aspirate and the severity of pneumonitis forms the basis for "standard" prolonged fasting prior to anaesthesia.

Since the 1980s, the duration of fasting from fluids has been questioned. Use of preoperative fluids reduces thirst, hunger, anxiety, fatigue, and postoperative pain and can reduce the extent of insulin resistance that occurs in the perioperative period. ${ }^{2}$ Physiological studies show that small volumes of ingested fluids are emptied within an hour. ${ }^{2}$ Although the use of gastric volume, a surrogate outcome, limits the strength of the evidence, the sample size needed to show equivalence in aspiration rates between "shortened" and "standard" fast times is prohibitive. Current evidence from RCTs does not show increased gastric volumes with shortened fast times $(<4 \mathrm{~h}) i^{3}$ thus, in healthy patients having elective or semi-elective surgery without risk factors for delayed gastric distention or emptying, the evidence does not support fast times over 3 hours.

Peter T-L Choi, MD, MSc University of British Columbia Vancouver, British Columbia, Canada

1 Jenkins K, Baker AB. Consent and anaesthetic risk. Anaesthesia 2003;58:962-84.

2 Liungqvist O, Soreide E. Preoperative fasting. Br J Surg 2003;90:400-6.

3 Practice guidelines for preoperative fasting and the use of pharmacologic agents to reduce the risk of pulmonary aspiration: application to healthy patients undergoing elective procedures: a report by the American Society of Anesthesiologist Task Force on Preoperative Fasting. Anesthesiology 1999;90:896-905. 